\title{
Motion of an impurity particle in an ultracold quasi-one-dimensional gas of hard-core bosons
}

\author{
M. D. Girardeau光 and A. Minguzzi ${ }^{2}$, \\ ${ }^{1}$ College of Optical Sciences, University of Arizona, Tucson, AZ 85721, USA \\ ${ }^{2}$ Université Joseph Fourier, Laboratoire de Physique et Modélisation \\ des Mileux Condensés, C.N.R.S., B.P. 166, 38042 Grenoble, France
}

(Dated: October 26, 2018)

\begin{abstract}
The low-lying energy eigenstates of a one-dimensional (1D) system of many impenetrable point bosons and one moving impurity particle with repulsive zero-range impurity-boson interaction are found for all values of the impurity-boson mass ratio and coupling constant. The moving entity is a polaron-like composite object consisting of the impurity clothed by a co-moving gray soliton. The special case with impurity-boson interaction of point hard-core form and impurity-boson mass ratio $m_{i} / m$ unity is first solved exactly as a special case of a previous Fermi-Bose (FB) mapping treatment of soluble 1D Bose-Fermi mixture problems. Then a more general treatment is given using second quantization for the bosons and the second-quantized form of the FB mapping, eliminating the impurity degrees of freedom by a Lee-Low-Pines canonical transformation. This yields the exact ground state (total linear momentum $q=0$ ) and exact boson-impurity distribution function in the thermodynamic limit for arbitrary $m_{i} / m$ and arbitrary impurity-boson interaction strength. These results are then extended to states with $q>0$.
\end{abstract}

PACS numbers: 03.75.-b, 67.85.-d

Due to the rapidly increasing sophistication of experimental techniques for probing ultracold gases, theoretical emphasis has shifted from effective field approaches to more refined methods capable of dealing with correlations. When confined in a de Broglie waveguide with transverse trapping so tight that longitudinal energies are less than the transverse vibrational excitation energy quantum $\hbar \omega_{\perp}$, an ultracold Bose gas becomes effectively one-dimensional (1D) with a confinement-induced resonance in the $1 \mathrm{D}$ scattering length [1]. In units such that $\hbar=1$, the dimensionless $1 \mathrm{D}$ coupling constant is $\gamma_{B}=m g_{1 D}^{B} / n$ where $g_{1 D}^{B}$ is the coupling constant for zero-range interactions $g_{1 D}^{B} \delta\left(x_{j}-x_{\ell}\right)$ [1] of Lieb-Liniger (LL) form [2], and $n$ is the $1 \mathrm{D}$ density $n=N / L$ with $N$ the number of bosons and $L$ the cell length for periodic boundary conditions. At low densities where $\gamma_{B} \gg 1$, the boson-boson interaction reduces to impenetrable point form, the so-called Tonks-Girardeau (TG) limit for which the exact many-body ground and excited states were found some 48 years ago by the Fermi-Bose (FB) mapping method [3]. The fermionization predicted therein has recently been experimentally confirmed [4, 5], leading to much recent theoretical and experimental activity on various properties of such strongly-correlated quasi$1 \mathrm{D}$ ultracold gases. It was found recently [6] that impurity atoms in an ultracold Bose gas with velocity $v_{i}$ have collision cross sections with the bosons which increase dramatically when $v_{i}>c$ where $c$ is the speed of sound in the Bose gas. Here we shall investigate the behavior of a moving impurity particle in an ultracold $1 \mathrm{D}$ gas of impenetrable point bosons, assuming a repulsive, zerorange impurity-boson interaction of arbitrary strength, and arbitrary impurity-boson mass ratio.
Hard-core impurity in a TG gas: Consider first the case $N_{F}=1$ of our previous exact solution of the problem of $N$ hard-core bosons and $N_{F}$ hard-core fermions in 1D [7]. One starts from a "model wavefunction" $\Psi_{M}=$ $\sum_{P} \varepsilon(P) u_{1}\left(P x_{1}\right) \cdots u_{N}\left(P x_{N}\right) u_{N+1}(y)$ where $y$ is the impurity position and the sum runs over all $(N+1)$ ! possible permutations of these variables including permutations exchanging bosons with the impurity, $\varepsilon(P)$ is the usual \pm 1 sign of the permutation, and $u_{1}, \cdots, u_{N+1}$ are $N+1$ orthonormal orbitals occupied by all $N$ bosons plus the one impurity. $\Psi_{M}$ vanishes at all the points $x_{j}=x_{\ell}$ and $x_{j}=y$ required by the hard-core constraints, and its improper symmetry under boson-boson and boson-impurity exchange can be repaired by a generalized FB mapping function $A=\prod_{1 \leq j<\ell \leq N} \operatorname{sgn}\left(x_{j}-x_{\ell}\right) \prod_{j=1}^{N} \operatorname{sgn}\left(x_{j}-y\right)[7]$ where the sign function $\operatorname{sgn}(x)$ is $+1(-1)$ if $x>0(x<$ $0)$. Then the physical wavefunction is $\Psi\left(x_{1}, \cdots, x_{N} ; y\right)=$ $A\left(x_{1}, \cdots, x_{N} ; y\right) \Psi_{M}\left(x_{1}, \cdots, x_{N} ; y\right)$. The model wave function $\Psi_{M}$ is an exact many-body energy eigenstate if the orbitals $u_{\nu}(x)$ are eigenfuctions of the single-freeparticle Hamiltonian $-\frac{1}{2 m} \frac{\partial^{2}}{\partial x^{2}}$ with eigenvalues $\epsilon_{\nu}$, and the physical state $\Psi=A \Psi_{M}$ is an energy eigenstate with eigenvalue $\sum_{\nu} \epsilon_{\nu}$. If there is no external potential and the system is on a ring of circumference $L$ with the $x_{j}$ and $y$ measured circumferentially, the $u_{\nu}$ are plane waves $L^{-1 / 2} e^{i k_{\nu} x}$ with $k_{\nu}$ which are integer $(+,-, 0)$ multiples of $\frac{2 \pi}{L}$ and $\epsilon_{\nu}=\frac{k_{\nu}^{2}}{2 m}$.

Assuming $N$ to be even, the model ground state $\Psi_{0 M}$ is a filled Fermi sea of the $N+1$ lowest plane-wave orbitals with $-k_{F} \leq k_{\nu} \leq k_{F}$ where $k_{F}=n \pi$ and $n=N / L$, i.e., $\nu=-\frac{N \pi}{L},-\frac{(N-2) \pi}{L}, \cdots, \frac{(N-2) \pi}{L}, \frac{N \pi}{L}$ and has total linear (or angular, on a ring) momentum zero. For this moving impurity problem, one needs excited states of nonzero to- 


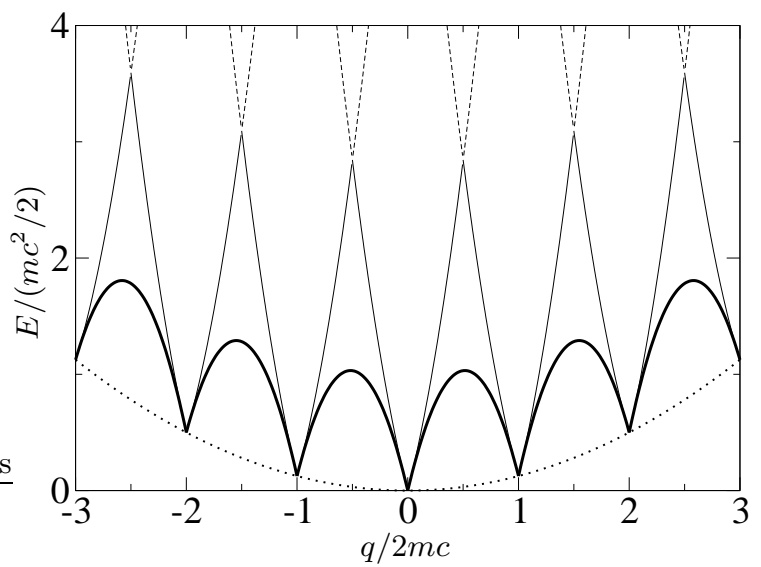

FIG. 1: Excitation energy branches as functions of the momentum transfer $q$ for $N+1=31$ particles. The bold solid lines indicate the lowest-energy (hole) excitations, the thinner solid lines indicate the lowest excitations of both Fermisurface (FS) and umklapp types, the dashed lines are the prolongations of the FS and umklapp excitations to higher-energy values, and the underlying dashed parabola is $q^{2} / 2(N+1) m$.

tal momentum $q$. The energy landscape is identical with that of $N+1$ TG bosons; only the physical interpretation is different. These excitations are the $\gamma_{B} \rightarrow+\infty$ limit of the types I and II excitations of the LL gas, Fig. 4, p. 1620 of [2], and are shown in Fig,1, The lowest excitations, indicated by the bold solid lines, correspond to the type II excitations of [2] and correspond to promoting a particle from $k_{F}-q+2 \pi / L$ to $k_{F}+2 \pi / L$ or from $-k_{F}+|q|-2 \pi / L$ to $-k_{F}-2 \pi / L$, thus leaving a hole in the interior of the Fermi sea. As $q$ increases from zero to $2 k_{F}$ the hole moves to the left from $k_{F}$ to $-k_{F}$, at which point the whole Fermi sea has been shifted to the right by $2 \pi / L$. Here $c=n h / 2 m$ is the speed of sound in the TG gas [3]. The excitation energy is periodic in the center of mass system with period $2 m c=2 k_{F}$ [9] , and the solid lines for $|q|>2 m c$ correspond to this periodicity, the underlying dashed parabola $q^{2} / 2(N+1) m$ being the result of transformation from the center of mass to the laboratory system, and the bold solid lines correspond to hole excitations from the original and displaced Fermi surfaces $k_{F}+j 2 \pi / L$ and $-k_{F}+j 2 \pi / L$ with $j=0, \pm 1, \pm 2, \cdots$. When $j$ reaches $(N+1) / 2$ the flow speed $v_{j}=j 2 \pi / m L$ reaches the speed of sound $c$, the slope in the entire interval $q_{j-1}<q<q_{j}$ becomes $\geq 0$ so that the energy barrier disappears, metastability is lost, and Mach wave drag commences. The boson-impurity distribution function $\rho_{b i}(x-y)=N(N-1) \int\left|\Psi\left(x, x_{2}, \cdots, x_{N} ; y\right)\right|^{2} d x_{2} \cdots d x_{N}$ is found in the thermodynamic limit to be $\rho_{b i}(x-y)=$ $n^{2}\left[1-j_{0}^{2}\left(k_{f}(x-y)\right)\right]$ where $j_{0}(\xi)=\sin \xi / \xi$ is the spherical Bessel function of order zero. This is independent of the flow speed $v_{j}$ and agrees with [3].

Quantized field representation: Generalize now to the case where impurity and boson masses are unequal and boson-impurity interactions are of the form $\lambda_{b i} \delta\left(x_{j}-\right.$ y). The impenetrable point boson-boson interaction will eventually be used, but initially assume it to be $\lambda_{b} \delta\left(x_{j}-x_{\ell}\right)$. The method used is motivated by a previous 3D weak-coupling treatment [8]. We use second quantization for the bosons while retaining Schrödinger representation for the impurity, with position variable $y$ and momentum operator $\hat{p}_{i}=\frac{1}{i} \frac{\partial}{\partial y}$. Then the Hamiltonian is

$$
\begin{gathered}
\hat{H}_{B}=\int d x \hat{\psi}_{B}^{\dagger}(x)\left[-\frac{1}{2} \frac{\partial^{2}}{\partial x^{2}}\right] \hat{\psi}_{B}(x) \\
+\frac{\lambda_{b}}{2} \int d x\left[\hat{\psi}_{B}^{\dagger}(x)\right]^{2} \hat{\psi}_{B}^{2}(x)-\frac{1}{2 m_{i}} \frac{\partial^{2}}{\partial y^{2}}+\lambda_{b i} \hat{\rho}_{B}(y)(1)
\end{gathered}
$$

where $\hat{\psi}_{B}(x)$ and $\hat{\psi}_{B}^{\dagger}(x)$ are the Bose field annihilation and creation operators, $\hat{\rho}_{B}(y)=\hat{\psi}_{B}^{\dagger}(y) \hat{\psi}_{B}(y)$ is the boson density operator at the impurity position, and we assume units wherein the boson mass $m=1$. Now enlarge the state space to also contain Fock states with Fermi field creation operators $\hat{\psi}_{F}^{\dagger}(x)$ where $\hat{\psi}_{F}$ and $\hat{\psi}_{F}^{\dagger}$ satisfy the usual anticommutation relations, but commute with the Bose fields, and both Bose and Fermi fields commute with the impurity position and momentum operators. On this enlarged Hilbert space, introduce a canonical transformation which is the second-quantized form [10] of the FB mapping [3]:

$$
\hat{\psi}_{B}(x)=e^{i \pi \int_{-\infty}^{x} d x^{\prime} \hat{\rho}_{B}\left(x^{\prime}\right)} \hat{\psi}_{F}(x)
$$

Then $\hat{H}_{B}$ is transformed into

$$
\hat{H}_{F}=\int d x \hat{\psi}_{F}^{\dagger}(x)\left[-\frac{1}{2} \frac{\partial^{2}}{\partial x^{2}}\right] \hat{\psi}_{F}(x)-\frac{1}{2 m_{i}} \frac{\partial^{2}}{\partial y^{2}}+\lambda_{b i} \hat{\rho}_{F}(y)
$$

where $\hat{\rho}_{F}(y)=\hat{\psi}_{F}^{\dagger}(y) \hat{\psi}_{F}(y)$ is the fermion density operator at the impurity position. The zero-range bosonboson interaction term in $\hat{H}_{B}$ has disappeared because its transform involves $\hat{\psi}_{F}^{2}(x)$ and its hermitian conjugate, which vanish by the anticommutation relations. Because of nonlocality of the FB mapping transformation, the transform of the boson kinetic energy operator does not have the simple form above on the whole Hilbert space, but on the subspace of states $\left|\Phi_{B}\right\rangle$ satisfying the impenetrable point hard core constraint $\hat{\psi}_{B}^{2}(x)\left|\Phi_{B}\right\rangle=0$ it reduces to this form [3, 10].

Next we make a second canonical transformation of the type used by Lee, Low, and Pines (LLP) in their theory of the polaron [11] which takes advantage of total linear momentum conservation so as to eliminate the impurity dynamical variables, as in [8]. It is effected by a unitary operator $\hat{U}=e^{-i y \hat{p}_{F}}$ where $\hat{p}_{F}=\int d x \hat{\psi}_{F}^{\dagger}(x) \frac{1}{i} \frac{\partial}{\partial x} \hat{\psi}_{F}(x)$ is the fermion momentum operator, with the result

$\hat{U}^{-1} y \hat{U}=y, \hat{U}^{-1} \hat{p}_{i} \hat{U}=\hat{p}_{i}-\hat{p}_{F}, \hat{U}^{-1} \hat{\psi}_{F}(x) \hat{U}=\hat{\psi}_{F}(x-y)$.

Then (3) is transformed into

$$
\hat{\mathcal{H}}=\hat{U}^{-1} \hat{H}_{F} \hat{U}=\hat{T}_{F}+\lambda_{b i} \hat{\rho}_{F}(0)+\frac{\left(\hat{p}_{i}-\hat{p}_{F}\right)^{2}}{2 m_{i}}
$$


where $\hat{T}_{F}$, the fermion kinetic energy operator, is the first term of Eq. (3). Since $y$ is absent from $\hat{\mathcal{H}}, \hat{p}_{i}$ commutes with $\hat{\mathcal{H}}$ and may be replaced by its eigenvalue $q$. Any energy eigenstate of $\hat{\mathcal{H}}$ with eigenvalue $E$ can be written as a direct product $|\Phi\rangle=L^{-1 / 2} e^{i q y}\left|\Phi_{F}\right\rangle$ where $\left|\Phi_{F}\right\rangle$ is independent of $y$. Then defining $\left|\Phi_{q}\right\rangle=\hat{U}|\Phi\rangle$ one finds that $\hat{H}_{F}\left|\Phi_{q}\right\rangle=E_{q}\left|\Phi_{q}\right\rangle$ and $\hat{P}\left|\Phi_{q}\right\rangle=q\left|\Phi_{q}\right\rangle$ provided that $\hat{\mathcal{H}}_{q}\left|\Phi_{q}\right\rangle=E_{q}\left|\Phi_{q}\right\rangle$, where $E_{q}$ is the energy eigenvalue of the given direct product eigenstate of $\hat{\mathcal{H}}, \hat{P}=\hat{p}_{i}+\hat{p}_{F}$ is the conserved total linear momentum (impurity plus fermions), and $\hat{\mathcal{H}}_{q}$ is the Hamiltonian obtained from $\hat{\mathcal{H}}$ by replacing the impurity momentum operator $\hat{p}_{i}$ by the c-number $q$. With this replacement Eq. (5) becomes

$$
\hat{\mathcal{H}}_{q}=\hat{T}_{F}+\lambda_{b i} \hat{\rho}_{F}(0)+\frac{q^{2}}{2 m_{i}}-\frac{q \hat{p}_{F}}{m_{i}}+\frac{\hat{p}_{F}^{2}}{2 m_{i}} .
$$

After mapping back to the physical Hilbert space (bosons plus impurity), $q$ is found to be the total linear momentum in the laboratory frame. The ground state has $q=0$, in which case two of the last three terms in (6) vanish identically. We will consider that case now and will generalize later to the case $q>0$. For $q=0$ the mean fermion momentum $\left\langle\hat{p}_{F}\right\rangle$ vanishes by symmetry, so the expectation value of the last term in (6) is proportional to the mean square fluctuation of $\hat{p}_{F}$. This fluctuation term will be shown later to be negligible in the thermodynamic limit, and will be dropped now.

Orbitals: With the last two terms dropped and the zero of energy shifted by $q^{2} / 2 m_{i}$, Eq. ([6]) is the Hamiltonian of free fermions in the field of a potential $\lambda_{b i} \delta(x)$ centered on the impurity. Its $N$-particle ground state is a filled Fermi sea of the lowest $N$ orbitals $\phi_{k}(x)$ of the singleparticle Schrödinger equation $\hat{H} \phi_{k}(x)=\epsilon_{k} \phi_{k}(x)$ with

$$
\hat{H}=-\frac{1}{2} \frac{\partial^{2}}{\partial x^{2}}+\lambda_{b i} \delta_{\text {per }}(x)
$$

where $\delta_{\text {per }}$ is an $L$-periodicized delta function, $\delta_{\text {per }}(x)=$ $\sum_{\nu=-\infty}^{\infty} \delta(x+\nu L)$. Our approach here is a generalization of a previous treatment of dark solitons in a TG gas [12]. As in [3] we assume that $N$ is odd so that the mapping back to the boson Hilbert space will be periodic. It is convenient to choose real eigenstates of (7) since the $\mathrm{N}$ fermion ground state has zero total linear momentum, and this will be satisfied automatically if all the orbitals have zero flux. We generalize Eq. (6) of [12] to

$$
\begin{aligned}
& \phi_{k}^{(+)}(x)=\mathcal{N}_{k}\left[\sin (k|x|)+A_{k} \cos (k x)\right], \\
& \phi_{k}^{(-)}(x)=\sqrt{2 / L} \sin (k x)
\end{aligned}
$$

where the allowed $k$-values for both the even-parity orbitals $\phi_{k}^{(+)}$and the odd-parity orbitals $\phi_{k}^{(-)}$are $k=$ $2 \pi / L, 4 \pi / L, \cdots$. These orbitals satisfy periodic boundary conditions on the interval $-L<x<L$, and are to be periodically extended to all other periodicity cells 13 . The delta function potential induces cusps in the even orbitals $\phi_{k}^{(+)}$at $x=0$ and more generally $x$ which are integral multiples of $L$, which are to be determined from $\phi_{k}{ }^{\prime}(0+)-\phi_{k}{ }^{\prime}(0-)=2 \phi_{k}{ }^{\prime}(0+)=2 \lambda_{b i} \phi_{k}(0)$ where the prime denotes the derivative, thus determining $A_{k}$ and hence the normalization constant $A_{k}$. One finds $A_{k}=$ $k / \lambda_{b i}$ and $\mathcal{N}_{k}=\sqrt{2 / L\left(1+A_{k}^{2}\right)}=\lambda_{b i} \sqrt{2 / L\left(\lambda_{b i}^{2}+k^{2}\right)}$. As in [12], the odd orbitals $\phi_{k}^{(-)}$do not see the delta potential and are cusp-free.

Impurity-boson distribution function: By Eq. (4), the impurity-boson distribution function $\rho_{b i}$ in the laboratory system is the expectation value of $\hat{\psi}_{F}^{\dagger}(x-y) \hat{\psi}_{F}(x-y)$ where $y$ is the impurity position. Then $\rho_{b i}(x-y)=$ $\sum_{\text {occ }}\left|\phi_{k}(x-y)\right|^{2}$ where $\sum_{\text {occ }}$ runs over the $N$ occupied orbitals with $\frac{2 \pi}{L} \leq k \leq(N+1) \pi / L$. By Eq. (4) and the argument following Eq. (5), the wave function in the laboratory system is $\Phi_{\text {lab }}\left(x_{1}, \cdots, x_{N} ; y\right)=$ $L^{-1 / 2} e^{i q y} \Phi_{F}\left(x_{1}-y, \cdots, x_{N}-y\right)$ where $\Phi_{F}$ is the filled Fermi sea. All of the linear momentum resides in the prefactor $e^{i q y}$ since the factor $\Phi_{F}$ is now translationally invariant. Application of the standard definition $\rho_{b i}(x-$ $y)=N(N-1) \int\left|\Phi_{\text {lab }}\left(x, x_{2}, \cdots, x_{N} ; y\right)\right|^{2} d x_{2} \cdots d x_{N}$ verifies the correctness of the above expression for $\rho_{b i}$ as a sum over density contributions from all occupied orbitals. In the thermodynamic limit the sum over the trivial orbitals with $A_{k}=0$ is an integral over the Fermi sea from 0 to $k_{F}$, yielding $\frac{n}{2}\left[1-j_{0}\left(2 k_{F}(x-y)\right)\right]$ where $j_{0}(z)=\sin (z) / z$ and $n=\frac{N}{L}=\frac{k_{F}}{\pi}$ is the mean fermion number density. In the limiting cases $\tilde{\lambda}_{b i}=\lambda_{b i} / k_{F}=0$ and $\tilde{\lambda}_{b i} \rightarrow \infty$ the sum over the orbitals with $A_{k} \neq 0$ can also be evaluated analytically in the thermodynamic limit; combining with the above contribution one finds $\rho_{b i}(x-y)=k_{F} / \pi=n$ for $\tilde{\lambda}_{b i}=0$ as expected, and for $\tilde{\lambda}_{b i} \rightarrow \infty$ (impenetrable impurity) one finds $\rho_{b i}(x-y)=$ $n\left[1-j_{0}\left(2 k_{F} x\right)\right]$ which vanishes when $x-y=0$ as expected. For intermediate values of $\tilde{\lambda}_{b i}$ the sum over the $\frac{N+1}{2}=200$ lowest orbitals with $A_{k} \neq 0$ has been evaluated numerically, and adding the above expression for the $A_{k}=0$ contribution yields the results shown in Fig. 2 for $\rho_{b i}(x-y)$. These results differs from the first section (one-fermion case of the boson-impurity mixture theory 7]) because the many-body wave function there represented the impurity "dissolved" into the TG gas with the total linear momentum $q$ shared equally by the impurity and $N$ bosons, whereas here the impurity moves through a nonmoving sea of fermions.

Generalization to $q>0$ : Here we generalize to the case of a moving impurity by including the term $-\frac{q \hat{p}_{F}}{m_{i}}$ in Eq. (6). Dropping $\hat{p}_{F}^{2} / 2 m_{i}$ and the c-number $\frac{q^{2}}{2 m_{i}}$, one finds that the $N$-particle ground state is a filled Fermi sea of the lowest $N$ orbitals $\phi_{k}(x)$ of the single-particle Schrödinger equation $\hat{H}_{q} \phi_{k}(x)=\left(k^{2} / 2\right) \phi_{k}(x)$ with

$$
\hat{H}_{q}=\frac{1}{2}\left(\frac{1}{i} \frac{\partial}{\partial x}-Q\right)^{2}+\lambda_{b i} \delta_{\mathrm{per}}(x)
$$

where $Q=q / m_{i}$. The momentum shift $Q$ can be 


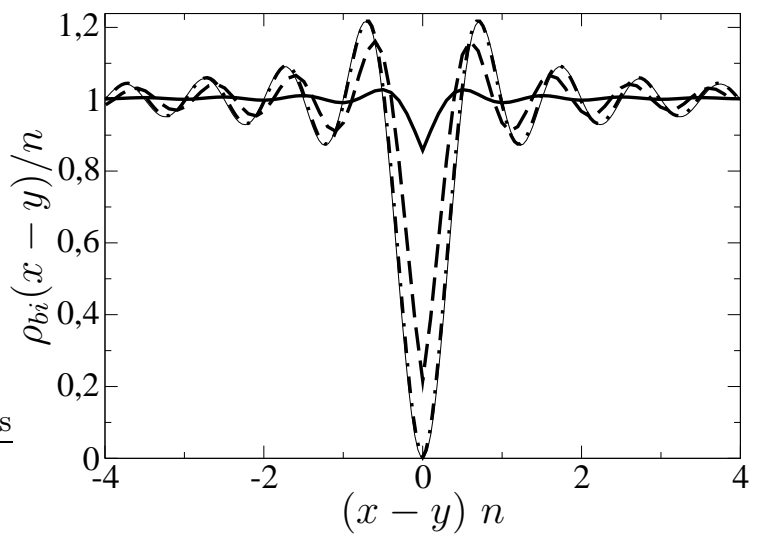

FIG. 2: Impurity-boson distribution function for various choices of impurity-boson coupling constant $\tilde{\lambda}_{b i}$. Thin solid line: $\tilde{\lambda}_{b i}=\infty$ from analytical expression. Other curves from numerical calculations with $\frac{N+1}{2}=200$ orbitals: thick solid line $\tilde{\lambda}_{b i}=0.1$, dashed line $\tilde{\lambda}_{b i}=1$, dot-dashed line $\tilde{\lambda}_{b i}=10$.

eliminated by a gauge transformation, writing $\phi_{k}(x)=$ $e^{i Q x} u_{k}(x)$, after which $u_{k}$ is an eigenfunction of the original Schrödinger equation (7). However, now periodicity of $\phi_{k}$ is not automatic, but must be enforced, leading to nontrivial allowed values of $k$ determined by a secular equation. This greatly complicates the solution. However, simple results can be obtained in the thermodynamic limit by noting that the total momentum $q$ is necessarily an integral multiple of $2 \pi / L$, i.e., $q=\nu 2 \pi / L$ with $\nu$ a non-negative integer. For finite, nonzero $q$, the thermodynamic limit is then to be attained by letting the integer $\nu \rightarrow \infty$ and fermion number $N \rightarrow \infty$ in such a way that $\nu / N \rightarrow q / 2 \pi n$ where $n=N / L$ is the fermion density in the thermodynamic limit where $N \rightarrow \infty, L \rightarrow \infty$, $N / L \rightarrow n$. In that limit there are infinitely many values of $m_{i}$ which are divisors of $\nu=L q / 2 \pi$, and the set of all such divisors is dense on the positive real axis. For all such $m_{i}, Q$ is an integral multiple of $2 \pi / L$, the orbitals reduce to the $q=0$ orbitals (8), the mean fermion momentum $\left\langle\hat{p}_{F}\right\rangle=0$, and the impurity-boson distribution function reduces to that of Fig. 2

Momentum fluctuation and thermodynamic limit: Here we show that the term $\frac{\hat{p}_{F}^{2}}{2 m_{i}}$ in (6) is negligible. $\left\langle\hat{p}_{F}\right\rangle$ is zero since the orbitals $\left.(8)_{i}\right)$ are real and have zero flux; hence $\left\langle\hat{p}_{F}^{2}\right\rangle=\left\langle\Psi_{0}\left|\hat{p}_{F}^{2}\right| \Psi_{0}\right\rangle$ is the mean square fluctuation of the fermion momentum about its mean. The $N$ fermion ground state is $\left|\Psi_{0}\right\rangle=\left(\prod_{k} \hat{c}_{k}^{\dagger}\right)|0\rangle$ where the $\hat{c}_{k}^{\dagger}$ are creation operators for the $N$ lowest orbitals $\phi_{k}$ of Eq. (8). One has $\hat{p}_{F}=\sum_{k k^{\prime}} \hat{c}_{k}^{\dagger}\left(\phi_{k}\left|\frac{1}{i} \frac{\partial}{\partial x}\right| \phi_{k^{\prime}}\right) \hat{c}_{k^{\prime}}$. The diagonal elements $\left(\phi_{k}^{(+)}\left|\frac{1}{i} \frac{\partial}{\partial x}\right| \phi_{k}^{(+)}\right)$and $\left(\phi_{k}^{(-)}\left|\frac{1}{i} \frac{\partial}{\partial x}\right| \phi_{k}^{(-)}\right)$vanish since their integrands are odd, and the off-diagonal elements lead to $\hat{p}_{F}=\sum_{k}\left[\frac{-i k A_{k}}{2 \sqrt{1+A_{k}^{2}}} \hat{c}_{k}^{(+) \dagger} \hat{c}_{k}^{(-)}+\right.$h.c. $]+$ $\sum_{k k^{\prime}}\left[\mathcal{O}\left(L^{-1}\right) \hat{c}_{k}^{(+) \dagger} \hat{c}_{k^{\prime}}^{(-)}+\right.$h.c.] where $\mathcal{O}\left(L^{-1}\right)$ is a function of $k$ and $k^{\prime}$ which is proportional to $L^{-1}$ in the thermodynamic limit where $N \rightarrow \infty$ and $L \rightarrow \infty$ with $n=N / L$ fixed. The $\sum_{k}$ vanishes when acting on $\left|\Psi_{0}\right\rangle$, since both $\phi_{k}^{(+)}$and $\phi_{k}^{(-)}$are occupied below the Fermi surface and both empty above it. There are two factors of $L^{-1}$ from the other term and two factors of $L / 2 \pi$ from $\sum_{k k^{\prime}} \rightarrow(L / 2 \pi)^{2} \int d k d k^{\prime}$, so $\frac{1}{2 m_{i}}\left\langle\Psi_{0}\left|\hat{p}_{F}^{2}\right| \Psi_{0}\right\rangle=\mathcal{O}(1)$ in the thermodynamic limit, negligible compared with the total ground state energy which is $\mathcal{O}(L)$. We expect that the contribution of this term to $\rho_{b i}(x-y)$ is only $\mathcal{O}\left(L^{-1}\right)$. The mean square fluctuation of the impurity momentum $\hat{p}_{i}$ is also $\mathcal{O}(1)$ since the total momentum $q$ is conserved.

Prospects: We have obtained exact results in the thermodynamic limit for the ground state energy and impurity-boson distribution function of an impurity in a TG gas, for arbitrary impurity-boson mass ratio and interaction strength. Control of these two additional parameters should provide an arena for future experiments building on the pathbreaking experiments [4, 5].

The first part of this paper is an outgrowth of work 7] initiated when we were participants in the 2007 workshop "Quantum Gases" at the Institut Henri Poincaré-Centre Emile Borel (IHP) in Paris, and we are grateful to the workshop organizers and the IHP for hospitality and support. We also thank Ewan Wright for helpful comments. Research of A.M. is supported by the Centre National de la Recherche Scientifique (CNRS).

* Electronic address: girardeau@optics.arizona.edu

$\dagger$ Electronic address: anna.minguzzi@grenoble.cnrs.fr

[1] M. Olshanii, Phys. Rev. Lett. 81, 938 (1998).

[2] E.H. Lieb and W. Liniger, Phys. Rev. 130, 1605 (1963).

[3] M. Girardeau, J. Math. Phys. 1, 516 (1960); M.D. Girardeau, Phys. Rev. 139, B500 (1965), Secs. 2, 3, 6.

[4] B. Paredes, et al., Nature 429, 277 (2004); T. Kinoshita, T.R. Wenger, and D.S. Weiss, Science 305, 1125 (2004).

[5] T. Kinoshita, T.R. Wenger, and D.S. Weiss, Phys. Rev. Lett. 95, 190406 (2005) and Nature 440, 900 (2006).

[6] A.P. Chikkatur, A. Görlitz, D.M. Stamper-Kurn, S. Inouye, S. Gupta, and W. Ketterle, Phys. Rev. Lett. 85, 483 (2000).

[7] M.D. Girardeau and A. Minguzzi, Phys. Rev. Lett. 99, 230402 (2007).

[8] M. Girardeau, J. Phys. Fluids 4, 279 (1961).

[9] F. Bloch, Phys. Rev. A 7, 2187 (1973).

[10] D.M. Gangardt and M. Pustilnik, Phys. Rev. A 77, 041604(R) (2008).

[11] T.D. Lee, F.E. Low, and D. Pines, Phys. Rev. 90, 297 (1953); M.D. Girardeau, Phys. Rev. A 28, 3635 (1983).

[12] M.D. Girardeau and E.M. Wright, Phys. Rev. Lett. 84, 5691 (2000).

[13] The periodicity interval for $\sin (k|x|)$ is $[-L, L]$ rather than $\left[-\frac{L}{2}, \frac{L}{2}\right]$ because of the absolute value; nevertheless, the orbitals (8) are orthonormal on $\left[-\frac{L}{2}, \frac{L}{2}\right]$, and both $\frac{1}{i} \frac{\partial}{\partial x}$ and the Hamiltonian (7) are hermitian on this interval, so we evaluate inner products on this interval. 